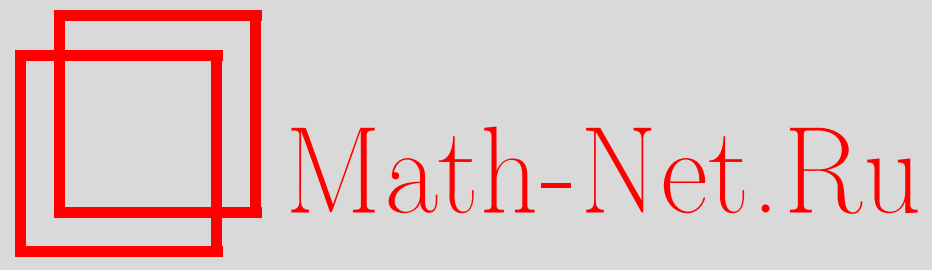

А. А. Усов, Способ решения краевых задач с неявным применением рядов Тейлора, Вестн. Сам. гос. техн. ун-та. Сер. Физ.-мат. науки, 2012, выпуск 4(), 227232

DOI: https://doi.org/10.14498/vsgtu1124

Использование Общероссийского математического портала Math-Net.Ru подразумевает, что вы прочитали и согласны с пользовательским соглашением

http: //www.mathnet.ru/rus/agreement

Параметры загрузки:

IP : 35.173 .137 .237

26 апреля 2023 г., 12:18:03

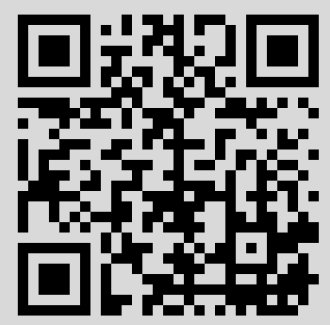


Вестн. Сам. гос. техн. ун-та. Сер. Физ.-мат. науки. 2012. № 4 (29). С. 227-232

УДК 517.927:519.624

\title{
СПОСОБ РЕШЕНИЯ КРАЕВЫХ ЗАДАЧ С НЕЯВНЫМ ПРИМЕНЕНИЕМ РЯДОВ ТЕЙЛОРА
}

\author{
A. A. Усов \\ Самарский государственный технический университет, \\ 443100, Россия, Самара, ул. Молодогвардейская, 244. \\ E-mail: ktmz@samgtu.ru
}

Предложен сеточный метод решения краевых задач для уравнений в частных производных на основе тейлоровских разложений высокого порядка, выполнено сравнение предложенного метода с классическим методом сеток. Показано, что использование разложения Тейлора с учётом заданного дифберенииального уравнения в частных производных позволяет существенно уменъшить погрешность численного решения при заданной неизменной дискретизации области за счёт увеличения порядка разложения. Решён ряд моделъных краевых задач, приводятся результаты оценки погрешности решения.

Ключевые слова: уравнения в частных производных, краевая задача, разложение Тейлора, метод сеток, аппроксимаиия, погрешность.

В работе [1] предложен неклассический метод численного решения краевых задач, основанный на применении рядов Тейлора со старшими производными, порядок которых выше порядка старшей производной заданного дифференциального уравнения. В работе [2] этот метод был применён к решению краевых задач для уравнения Лапласа, а в [3,4] он развит для решения обыкновенных дифференциальных уравнений, где исследованы вопросы сходимости и устойчивости метода. Данная работа является дальнейшим развитием указанных работ.

Пусть в области $D$ с кусочно-гладкой границей $\Gamma$ задано уравнение

$$
\begin{aligned}
L[u(x, y)] \equiv q_{0}(x, y) u+q_{1}(x, y) u_{x}+q_{2}(x, y) & u_{y}+q_{3}(x, y) u_{x x}+ \\
& +q_{4}(x, y) u_{x y}+q_{5}(x, y) u_{y y}=F(x, y),
\end{aligned}
$$

где $L$ - линейный дифференциальный оператор, $u(x, y)$ - искомая неизвестная функция, $q_{i}(i=0,1, \ldots, 5), F$ - дифференцируемые необходимое число раз заданные функции. Не касаясь всей широты и строгости задачи, рассмотрим предлагаемый метод на примере краевой задачи для уравнения (1) в частных производных, при этом граничные условия пока формулировать не будем. Выполним дискретизацию области по переменным $x$ и $y$. Обозначим $\Delta x_{i k}=x_{i}-x_{k}(i, k=\overline{1, n})$ и $\Delta y_{j s}=y_{j}-y_{s}$ $(j, s=\overline{1, m})$ соответственно. Рассмотрим шаблон, представленный на рис. 1.

$1^{\circ}$. Разложим в ряд Тейлора функцию $u(x, y)$ в точке $\left(x_{i}, y_{j}\right)$, ограничившись членами со второй производной:

$$
\begin{aligned}
u\left(x_{i}, y_{j}\right)+\Delta x_{k i} u_{x}\left(x_{i}, y_{j}\right) & +\Delta y_{s j} u_{y}\left(x_{i}, y_{j}\right)+\frac{\left(\Delta x_{k i}\right)^{2}}{2} u_{x x}\left(x_{i}, y_{j}\right)+ \\
+ & \Delta x_{k i} \Delta y_{s j} u_{x y}\left(x_{i}, y_{j}\right)+\frac{\left(\Delta y_{s j}\right)^{2}}{2} u_{y y}\left(x_{i}, y_{i}\right)=u\left(x_{k}, y_{s}\right),
\end{aligned}
$$

Александр Александрович Усов, инженер, каф. высшей математики и прикладной информатики. 


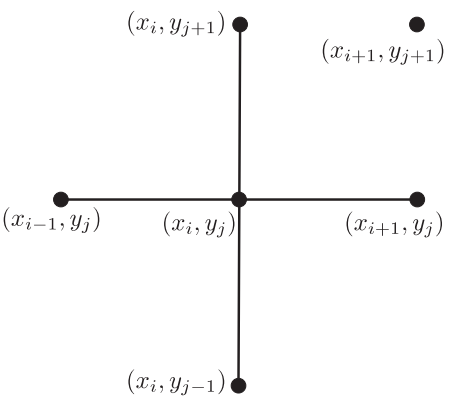

Рис. 1
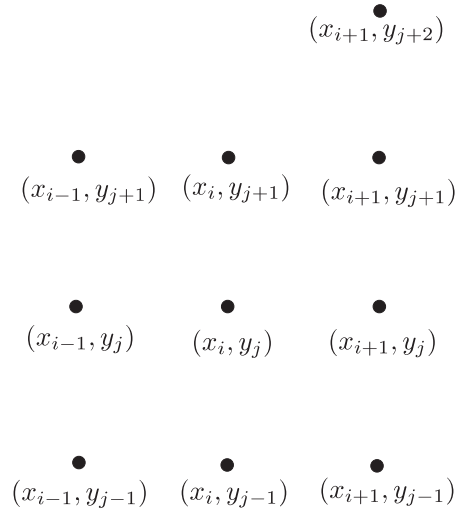

Рис. 2

где пары индексов $(k, s)$ в соответствии с шаблоном принимают следующие значения:

$$
(k, s)=\{(i, j+1),(i+1, j+1),(i+1, j),(i, j-1),(i-1, j)\} .
$$

С учётом изменения индексов соотношение (2) представляет систему пяти уравнений. Запишем (1) в точке $\left(x_{i}, y_{j}\right)$ :

$$
\begin{aligned}
& q_{0}\left(x_{i}, y_{j}\right) u\left(x_{i}, y_{j}\right)+q_{1}\left(x_{i}, y_{j}\right) u_{x}\left(x_{i}, y_{j}\right)+q_{2}\left(x_{i}, y_{j}\right) u_{y}\left(x_{i}, y_{j}\right)+ \\
& \quad+q_{3}\left(x_{i}, y_{j}\right) u_{x x}\left(x_{i}, y_{j}\right)+q_{4}\left(x_{i}, y_{j}\right) u_{x y}\left(x_{i}, y_{j}\right)+q_{5}\left(x_{i}, y_{j}\right) u_{y y}\left(x_{i}, y_{j}\right)=F\left(x_{i}, y_{j}\right)
\end{aligned}
$$

Объединяя пять уравнений (2) и (1) и считая значения функции и её производных в точке $\left(x_{i}, y_{j}\right)$ неизвестными, получим систему шести линейных уравнений относительно шести неизвестных. Обозначим через $p_{k s i j}^{(2)}$ вектор-строку коэффициентов при неизвестной функции $u$ и её производных в разложении (2) (верхний индекс здесь означает максимальный порядок частных производных, используемых в разложении Тейлора):

$$
p_{k s i j}^{(2)}=\left(1, \Delta x_{k i}, \Delta y_{s j}, \frac{\left(\Delta x_{k i}\right)^{2}}{2}, \Delta x_{k i} \Delta_{s j}, \frac{\left(\Delta y_{s j}\right)^{2}}{2}\right),
$$

где индексы $k$ и $s$ принимают значения (3).

Через $l$ обозначим вектор-строку, составленную из функций $q_{m}\left(x_{i}, y_{j}\right)(m=$ $=0,1, \ldots, 5)$ :

$$
l=\left(q_{0}, q_{1}, \ldots, q_{5}\right) .
$$

Введём в рассмотрение матрицу $M_{6 \times 6}$, строками которой являются пять строк (4), а шестая её строка состоит из элементов вектор-строки (5), а также матрицы $X_{6 \times 1}$ и $B_{6 \times 1}$ следующим образом:

$$
M_{6 \times 6}=\left(\begin{array}{c}
p_{i i+1 i j}^{(2)} \\
p_{i+1 j+1 i j}^{(2)} \\
p_{i+1 j i j}^{(2)} \\
p_{i j-1 i j}^{(2)} \\
p_{i-1 j-1 i j} \\
l
\end{array}\right), \quad X=\left(\begin{array}{c}
u\left(x_{i}, y_{j}\right) \\
u_{x}\left(x_{i}, y_{j}\right) \\
u_{y}\left(x_{i}, y_{j}\right) \\
u_{x x}\left(x_{i}, y_{j}\right) \\
u_{x y}\left(x_{i}, y_{j}\right) \\
u_{y y}\left(x_{i}, y_{j}\right)
\end{array}\right), \quad B=\left(\begin{array}{c}
u\left(x_{i}, y_{j+1}\right) \\
u\left(x_{i+1}, y_{j+1}\right) \\
u\left(x_{i+1}, y_{j}\right) \\
u\left(x_{i}, y_{j-1}\right) \\
u\left(x_{i-1}, y_{j-1}\right) \\
F\left(x_{i}, y_{j}\right)
\end{array}\right) .
$$

Тогда система уравнений (1), (2) может быть записана в матричном виде

$$
M X=B .
$$


Если матрица $M$ является невырожденной, т.е. $\operatorname{det} M \neq 0$, то решение (6) имеет вид

$$
X=M^{-1} B .
$$

Обозначим коэффициенты обратной матрицы $M^{-1}$ через $m_{\alpha \beta}^{-1}(\alpha, \beta=1,2, \ldots, 6)$. Тогда первая строка матричного равенства (7) даёт следующее соотношение:

$$
\begin{aligned}
u\left(x_{i}, y_{j}\right) & -m_{11}^{-1} u\left(x_{i}, y_{j+1}\right)-m_{12}^{-1} u\left(x_{i+1}, y_{j+1}\right)- \\
& -m_{13}^{-1} u\left(x_{i+1}, y_{j}\right)-m_{14}^{-1} u\left(x_{i}, y_{j-1}\right)-m_{15}^{-1} u\left(x_{i-1}, y_{j-1}\right)=m_{16}^{-1} F\left(x_{i}, y_{j}\right) .
\end{aligned}
$$

Обозначим через $N$ общее количество внутренних узлов области $D$, ограниченной границей $\Gamma$, и пусть известно значение функции $u(x, y)$ на этой границе:

$$
\left.u(x, y)\right|_{\Gamma}=\varphi(x, y) .
$$

Тогда, записывая соотношение (8) в каждом внутреннем узле дискретизации, получим систему $N$ уравнений относительно стольких же неизвестных искомой функции $u=u(x, y)$ в этих узлах. При этом за счёт неравномерной сетки дискретизации по переменным $x$ и $y$ всегда можно добиться того, что граничные узлы точно будут принадлежать границе Г и значения функции в этих узлах будут определяться равенством (9) (эти члены в соотношениях (8) необходимо перенести в их правую часть).

$2^{\circ}$. Увеличить точность численного решения краевой задачи (1), (9) (не изменяя количества узлов сетки) можно увеличением количества членов в разложении Тейлора, учитывая частные производные более высокого (чем второй) порядка. При этом, если порядок старшей частной производной равен $n$, то число узлов шаблона равно $2 n+1$. Проиллюстрируем этот вариант на конкретном примере при $n=4$. Рассмотрим шаблон из 9 узлов, представленный на рис. 2. Запишем разложение функции $u(x, y)$ в точке $\left(x_{i}, y_{i}\right)$ до частных производных четвёртого порядка:

$$
\begin{gathered}
u\left(x_{i}, y_{j}\right)+\Delta x_{k i} u_{x}\left(x_{i}, y_{j}\right)+\Delta y_{s j} u_{y}\left(x_{i}, y_{j}\right)+\frac{\left(\Delta x_{k i}\right)^{2}}{2} u_{x x}\left(x_{i}, y_{j}\right)+ \\
+\Delta x_{k i} \Delta y_{s j} u_{x y}\left(x_{i}, y_{j}\right)+\frac{\left(\Delta y_{s j}\right)^{2}}{2} u_{y y}\left(x_{i}, y_{j}\right)+\frac{\left(\Delta x_{k i}\right)^{3}}{3 !} u_{x x x}\left(x_{i}, y_{j}\right)+ \\
+\ldots+\frac{\left(\Delta y_{s j}\right)^{4}}{4 !} u_{y y y}\left(x_{i}, y_{j}\right)=u\left(x_{k}, y_{s}\right),
\end{gathered}
$$

где пары индексов $(k, s)$ в соответствии с шаблоном принимают следующие значения: $(k, s)=\{(i+2, j+1),(i+1, j-1),(i+1, j),(i+1, j+1),(i, j-1),(i, j+1)$, $(i-1, j-1),(i-1, j),(i-1, j+1)\}$.

Таким образом, (10) представляют из себя 9 уравнений. Примем в качестве неизвестных функцию $u\left(x_{i}, y_{j}\right)$ и все её частные производные до четвертого порядка включительно в этой же точке. В итоге число неизвестных равно 15. Присоединим к системе девяти уравнений (10) шесть уравнений, полученных из (1) следующим образом: уравнение (1), а также уравнения, полученные дифференцированием (1) по переменным $x, y, x x, x y$ и $y y$, записываются в точке $\left(x_{i}, y_{i}\right)$ :

$$
\begin{aligned}
q_{0}\left(x_{i}, y_{j}\right) u\left(x_{i}, y_{i}\right)+q_{1}\left(x_{i}, y_{j}\right) u_{x}( & \left.x_{i}, y_{j}\right)+\ldots+ \\
& +q_{5}\left(x_{i}, y_{j}\right) u_{y y}\left(x_{i}, y_{j}\right)=F\left(x_{i}, y_{j}\right), \\
\ldots & \\
\rho_{0}\left(x_{i}, y_{j}\right) u\left(x_{i}, y_{j}\right)+\rho_{1}\left(x_{i}, y_{j}\right) u_{x} & \left(x_{i}, y_{j}\right)+\ldots+ \\
& +\rho_{14}\left(x_{i}, y_{j}\right) u_{y y y y}\left(x_{i}, y_{j}\right)=F_{y y}\left(x_{i}, y_{j}\right),
\end{aligned}
$$

где $\rho_{\alpha}(\alpha=0,1, \ldots, 14)$ - функции, зависящие от $q_{\beta}(\beta=0,1, \ldots, 5)$ и их частных производных. 
Объединим девять уравнений (10) и 6 уравнений (11) в одну систему и будем её рассмтаривать относительно 15 неизвестных $u\left(x_{i}, y_{j}\right)$ и всех её частных производных в точке $\left(x_{i}, y_{j}\right)$ до четвёртого порядка включительно. Тогда эта система также может быть записана в матричном виде (7), где элементами матрицы $M_{15 \times 15}$ являются коэффициенты при функции $u$ и её производных в системе (10), (11), а матрицы $X_{15 \times 1} B_{15 \times 1}$ имеют вид

$$
\begin{gathered}
X=\left(u\left(x_{i}, y_{j}\right), u_{x}\left(x_{i}, y_{j}\right), u_{y}\left(x_{i}, y_{j}\right), u_{x x}\left(x_{i}, y_{j}\right), u_{x y}\left(x_{i}, y_{j}\right), u_{y y}\left(x_{i}, y_{j}\right)\right. \\
u_{x x x}\left(x_{i}, y_{j}\right), u_{x x y}\left(x_{i}, y_{j}\right), u_{x y y}\left(x_{i}, y_{j}\right), u_{y y y}\left(x_{i}, y_{j}\right), u_{x x x x}\left(x_{i}, y_{j}\right), \\
\left.u_{x x x y}\left(x_{i}, y_{j}\right), u_{x x y y}\left(x_{i}, y_{j}\right), u_{x y y y}\left(x_{i}, y_{j}\right), u_{y y y y}\left(x_{i}, y_{j}\right)\right)^{\top}, \\
B=\left(u\left(x_{i+2}, y_{j+1}\right), u\left(x_{i+1}, y_{j-1}\right), u\left(x_{i+1}, y_{j}\right), u\left(x_{i+1}, y_{j+1}\right), u\left(x_{i}, y_{j-1}\right),\right. \\
u\left(x_{i}, y_{j+1}\right), u\left(x_{i-1}, y_{j-1}\right), u\left(x_{i-1}, y_{j}\right), u\left(x_{i-1}, y_{j+1}\right), F\left(x_{i}, y_{j}\right), \\
\left.F_{x}\left(x_{i}, y_{j}\right), F_{y}\left(x_{i}, y_{j}\right), F_{x x}\left(x_{i}, y_{j}\right), F_{x y}\left(x_{i}, y_{j}\right), F_{y y}\left(x_{i}, y_{j}\right)\right)^{\top} .
\end{gathered}
$$

Обозначим коэффициенты обратной матрицы $M^{-1}$ также через $m_{\alpha \beta}^{-1}(\alpha, \beta=\overline{1,15})$. Тогда первая строка матричного равенства (7) даёт следующее соотношение:

$$
\begin{aligned}
u\left(x_{i}, y_{j}\right)-m_{11}^{-1} u\left(x_{i+2}, y_{j+1}\right)-m_{12}^{-1} u\left(x_{i+1}, y_{j-1}\right)-\ldots & \\
& -m_{10}^{-1} u\left(x_{i-1}, y_{j+1}\right)=m_{110}^{-1} F\left(x_{i}, y_{j}\right)-\ldots-m_{115}^{-1} F_{y y}\left(x_{i}, y_{j}\right) .
\end{aligned}
$$

Записывая (12) для каждой внутренней точки области дискретизации, получим замкнутую систему уравнений для всех узлов сетки, причём учёт граничных условий осуществляется аналогично рассмотренному случаю разложения Тейлора до производных второго порядка.

Аналогично можно получить расчётную схему для любого порядка $n>4$ старшей производной в разложении Тейлора.

$3^{\circ}$. Исследуем теперь на конкретных примерах погрешность метода для различных значений порядка $n$ старшей производной в разложении Тейлора. Обозначим через $u^{(n)}\left(x_{i}, y_{j}\right)$ численные решения заданной краевой задачи в узле $\left(x_{i}, y_{j}\right)$, а через $u^{a}\left(x_{i}, y_{i}\right)$ - аналитическое решение этой же задачи в заданном узле сетки. В качестве оценки погрешности выберем следующую норму:

$$
\|\delta\|=\left[\frac{\sum i, j\left[u^{(n)}\left(x_{i}, y_{j}\right)-u^{a}\left(x_{i}, y_{j}\right)\right]^{2}}{\sum_{i, j}\left(u^{a}\left(x_{i}, y_{j}\right)\right)^{2}}\right]^{1 / 2} .
$$

ПримеР 1. Найти решение уравнения

$$
\frac{\partial^{2} u}{\partial x^{2}}+\frac{\partial^{2} u}{\partial y^{2}}=0
$$

в квадрате $0,3 \leqslant x \leqslant 0,9,0,3 \leqslant y \leqslant 0,9$, если $u(0,3 ; y)=\ln \left(0,09+y^{2}\right), u(x ; 0,3)=$ $=\ln \left(x^{2}+0,09\right), u(0,9 ; y)=\ln \left(0,81+y^{2}\right), u(x ; 0,9)=\ln \left(x^{2}+0,81\right)$.

Аналитическое решение данной задачи имеет вид $u^{a}(x, y)=\ln \left(x^{2}+y^{2}\right)$. Для численного решения использовалось разложение до $n=4$ с одинаковыми шагами $h$ дискретизации по осям $x$ и $y$. Расчёты выполнены при трёх значениях шага дискретизации: $h=0,2 ; h=0,1 ; h=0,05$. В результате применения изложенной выше методики получены следующие оценки погрешности (13): при $h=0,2$ величина $\|\delta\|_{h=0,2}=6,55 \cdot 10^{-5}$; при $h=0,1-\|\delta\|_{h=0,1}=9,1 \cdot 10^{-7}$ и при $h=0,05-\|\delta\|_{h=0,05}=$ $=1,25 \cdot 10^{-8}$. Нетрудно получить следующие результаты: $\|\delta\|_{h=0,2} /\|\delta\|_{h=0,1} \approx 72$, 
$\|\delta\|_{h=0,1} /\|\delta\|_{h=0,05} \approx 72,8$, откуда следует, что при $n=4$ погрешность метода имеет порядок о $\left(h^{6}\right)$. При использовании классического метода сеток [5] имеем порядок $\mathrm{o}\left(h^{2}\right)$.

ПримеР 2. Выполнить оценку погрешности метода для дифференциального уравнения (1) в области $D=\left\{(x, y): 25 \leqslant x^{2}+y^{2} \leqslant 169\right\}$, если $\left.u(x, y)\right|_{x^{2}+y^{2}=25}=$ $=\ln 25,\left.u(x, y)\right|_{x^{2}+y^{2}=169}=\ln 169$.

В данном примере было выполнено исследование при двух равных шагах дискретизации по осям $O x$ и $O y: h=1$ и $h=0,5$ и значениях $n=2, n=4$ и $n=6$.

При $n=2$ получены следующие оценки нормы (13): $\|\delta\|_{h=1}=1,47 \cdot 10^{-4}$, $\|\delta\|_{h=0,5}=3,8 \cdot 10^{-5}$, поэтому $\|\delta\|_{h=1} /\|\delta\|_{h=0,5} \approx 3,87$, откуда имеем погрешность метода близкой к о $\left(h^{2}\right)$.

При $n=4$ имеем $\|\delta\|_{h=1}=1,148 \cdot 10^{-5},\|\delta\|_{h=0,05}=8,27 \cdot 10^{-7},\|\delta\|_{h=1} /\|\delta\|_{h=0,5} \approx$ 13,85 , откуда погрешность метода в этом случае составляет величину порядка о $\left(h^{4}\right)$.

При $n=6$ имеем $\|\delta\|_{h=1}=3,6 \cdot 10^{-7},\|\delta\|_{h=0,5}=5,28 \cdot 10^{-9},\|\delta\|_{h=1} /\|\delta\|_{h=0,5}=70,8$, откуда погрешность метода о $\left(h^{6}\right)$.

Из данного примера следует, что не изменяя числа узлов $(h=\mathrm{const})$, за счёт увеличения порядка разложения Тейлора можно существенно увеличить точность расчётов (до нескольких порядков), в чём можно убедиться, сравнивая величины погрешности $\|\delta\|$ во втором примере, например, при $h=0,05$ и различных значенияx $n$.

\section{БИБЛИОГРАФИЧЕСКИЙ СПИСОК}

1. Усов A. А. Об одном подходе к численному решению краевых задач на основании формулы Тэйлора/ В сб.: Труды восъмой межвузовской конферениии. Часть 3: Дифференциальные уравнения и краевые задачи / Матем. моделирование и краев. задачи. Самара: СамГТУ, 1998. С. 99-102. [Usov A.A. On one approach to the numerical solution of boundary value problems on the basis of Taylor's formula / In: Proceedings of the Eighth Inter-University Conference. Part 3 / Matem. Mod. Kraev. Zadachi. Samara: Samara State Technical Univ., 1998. Pp. 99-102].

2. Усов А.А., Радченко В. П. Об одном подходе к решению краевых задач для уравнений математической физики с применением разложения Тейлора/ В сб.: Труды шестой Всероссийской научной конферениии с международным участием (1-4 июня 2009 г.). Часть 1: Математические модели механики, прочности и надёжности элементов конструкций / Матем. моделирование и краев. задачи. Самара: СамГТУ, 2009. С. 271-276. [Usov A. A., Radchenko V. P. On one approach to the solution of boundary value problems for equations of mathematical physics with use of Taylor expansions / In: Proceedings of the Sixth All-Russian Scientific Conference with international participation (1-4 June 2009). Part 1/ Matem. Mod. Kraev. Zadachi. Samara: Samara State Technical Univ., 2009. Pp. 271-276].

3. Радченко В. П., Усов А. А., Модификация сеточных методов решения линейных дифференциальных уравнений с переменными коэффициентами на основе тейлоровских разложений // Вестн. Сам. гос. техн. ун-та. Сер. Физ.-мат. науки, 2008. № 2(17). С. 60-65. [Radchenko V.P., Usov A.A. Modified grid method for solving linear differential equation equipped with variable coefficients based on Taylor series // Vestn. Samar. Gos. Tekhn. Univ. Ser. Fiz.-Mat. Nauki, 2008. no. 2(17). Pp. 60-65].

4. Павлова Г.А., Беляева И. В. Модификация метода сеток с использованием разложений Тейлора // Вестн. Сам. гос. техн. ун-та. Сер. Физ.-мат. науки, 2012. №3(28). C. 152-162. [Pavlova G. A., Belyaeva I. V. Modification of finite differences method with use of Taylor expansions // Vestn. Samar. Gos. Tekhn. Univ. Ser. Fiz.-Mat. Nauki, 2012. no. 3(28). Pp. 152-162].

5. Волков Е. А. Численные методы. М.: Лань, 2004. 256 с. [Volkov E. A. Numerical methods. Moscow: Lan', 2004. 256 pp.] 
MSC: 34B99

BOUNDARY VALUE PROBLEMS SOLVING METHOD WITH THE IMPLICIT USE OF THE TAYLOR EXPANSIONS

\section{A. A. Usov}

Samara State Technical University,

244, Molodogvardeyskaya st., Samara, 443100, Russia.

E-mail: ktmz@samgtu.ru

Grid method for boundary value problems solving for partial differential equations based on high order Taylor expansions is suggested. Comparison of the proposed method with classical grid method is implemented. It is shown that the use of the Taylor expansion with specified partial differential equations allows to reduce the estimated faulty proportion of the numerical solution for a given constant sampling area by increasing the order of the expansion. A number of model boundary value problems is solved, the results of the estimated faulty proportion are given.

Key words: partial differential equations, boundary value problem, Taylor expansion, the grid method, approximation, estimated faulty proportion.

Original article submitted 02/VI/2012; revision submitted 25/VIII/2012.

Alexandr A. Usov, Engineer, Dept. of Higher Mathematics and Applied Computer Science. 